\title{
Control of switching circuits using complete-cycle solution matrices
}

\author{
Damian Giaouris, Member, IEEE, Abdulmajed Elbkosh, Soumitro Banerjee, Senior Member, IEEE, Bashar \\ Zahawi Senior Member, IEEE, and Volker Pickert Member, IEEE
}

\begin{abstract}
The bifurcation behavior of switching power electronic converters can be altered by appropriately changing the switching manifold. This paper examines this bifurcation control strategy and proposes new ways in which it can be implemented. A detailed comparison which includes transients as well as steady state properties is also presented. This method can be applied to control any piecewise smooth system to stabilize a desired periodic orbit.
\end{abstract}

\section{INTRODUCTION}

$\mathbf{T}$ HE NONLINEAR behavior of the buck converter is a well known and studied phenomenon [1]-[4]. Controlling this pattern is a challenging topic as it is required to ensure a stable period one operation over a wide range and at the same time to be relatively simple and easy to implement in real applications. Some of the existing methods of controlling this behavior (OGY, Pyragas) may require high processing power.

A new simpler method have already been proposed by the authors [5], [6] that also provides a good understanding of the internal mechanisms that usually cause period doubling bifurcations. This method was also used to control the buck converter and it greatly extends the region where we have a stable response [6]. This method is based on the calculation of the Floquet multipliers which are the eigenvalues of the monodromy matrix. The monodromy matrix, $\mathbf{M}(T, 0)$, is the fundamental solution matrix (also called State Transition Matrix or STM) over one full cycle. One of the basic properties of the STM is that we can break the evolution of the system to as many time intervals as we require, [7], [8]:

$$
\mathbf{M}(T, 0)=\boldsymbol{\Phi}\left(T, t_{n}\right) \times \mathbf{\Phi}\left(t_{n}, t_{n-1}\right) \ldots \mathbf{\Phi}\left(t_{1}, 0\right)
$$

where $\boldsymbol{\Phi}\left(t_{k}, t_{m}\right)$ is the STM from $t=t_{m}$ until $t=t_{k}$. If the system is time invariant then the STM can be calculated by the exponential matrix of the Jacobian of the vector field for the duration of that time interval. On the other hand if the system is time varying then there is no universally acceptable analytical method and the usual choice is the numerical integration of a matrix differential equation [9]:

$$
\dot{\mathbf{\Phi}}\left(t, t_{0}\right)=\frac{\partial \mathbf{f}(t, \mathbf{x})}{\partial \mathbf{x}} \times \mathbf{\Phi}\left(t, t_{0}\right), \quad \mathbf{\Phi}\left(t_{0}, t_{0}\right)=\mathbf{I}
$$

D. Giaouris, A. Elbkosh B. Zahawi, and V. Pickert are with the School of Electrical, Electronic and Computer Engineering, University of Newcastle upon Tyne, UK. E-mail: Damian.Giaouris@ newcastle.ac.uk

S. Banerjee is with the Centre for Theoretical Studies and Department of Electrical Engineering, Indian Institute of Technology, Kharagpur-721302, India. E-mail: soumitro@iitkgp.ac.in

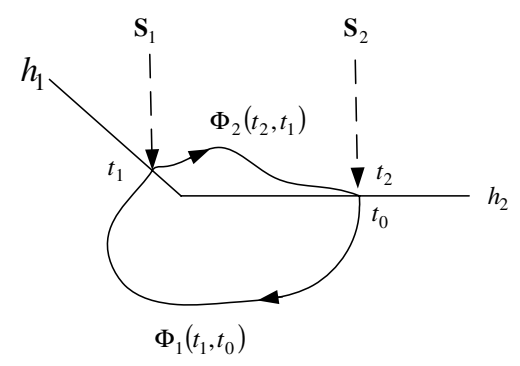

Fig. 1. General periodic orbit that crosses two switching manifolds

If the system is piecewise smooth (as is the case for most power electronic circuits) then we can break the monodromy matrix to areas where the vector field is smooth and to areas where the vector field crosses a switching manifold (Fig. 1):

$$
\mathbf{M}\left(T+t_{0}, t_{0}\right)=\mathbf{S}_{2} \times \boldsymbol{\Phi}_{2}\left(T+t_{0}, t_{1}\right) \times \mathbf{S}_{1} \times \mathbf{\Phi}\left(t_{1}, t_{0}\right)
$$

The STM during the switching is referred to as the saltation matrix $\mathbf{S}$. If the transition is from $f_{+}$to $f_{-}$(at $t=t_{\Sigma}$ )then $\mathbf{S}$ is given by:

$$
\mathbf{S}=\mathbf{I}+\frac{\left(\lim _{t \downarrow t_{\Sigma}}\left(\mathbf{f}_{-}(\mathbf{x}(t))\right)-\lim _{t \uparrow t_{\Sigma}}\left(\mathbf{f}_{+}(\mathbf{x}(t))\right)\right) \mathbf{n}^{T}}{\mathbf{n}^{T} \lim _{t \uparrow t_{\Sigma}}\left(\mathbf{f}_{+}(\mathbf{x}(t))\right)+\frac{\partial h}{\partial t}\left(\mathbf{x}(t), t_{\Sigma}\right)}
$$

The purpose of this paper is to propose a new control strategy based on the monodromy matrix for stabilizing various closed orbits of the system. We also present a thorough comparison between the various controllers. The application of the control strategy is also extended to another dc-dc converter, namely the boost converter.

\section{BUCK CONVERTER - STABILITY ANALYSIS}

The voltage controlled buck converter (Fig. 2) has been studied by many researchers [3], [10], [11] and its bifurcation structure is well known. The desired operation of this circuit is a limit cycle around a pre-defined value whose period equals the period of the ramp signal $\left(v_{\text {ramp }}\right)$. If the input voltage is changed then it is possible to have a period doubling bifurcation and hence a new limit cycle will exist with a period twice as the period of the ramp signal as shown in Fig. 3 .

To study the period one limit cycle we study the system in $t \in[0, T)$ so the switching manifold is smooth and is defined as:

$$
h(\mathbf{x}(t), t)=x_{1}(t)-V_{\mathrm{ref}}-\frac{V_{L}+\left(V_{U}-V_{L}\right) t / T}{A}=0
$$




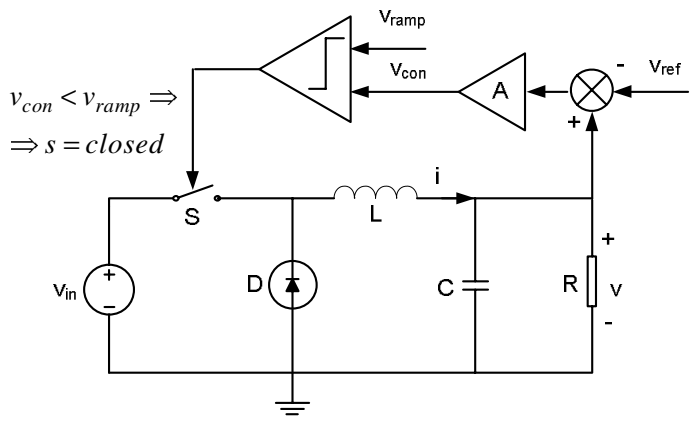

Fig. 2. The voltage mode controlled buck dc-dc converter.

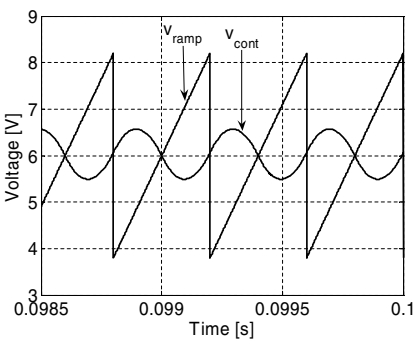

(a)

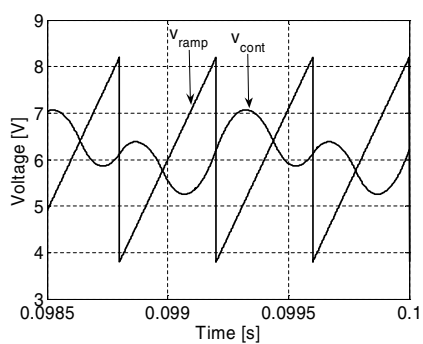

(b)
Fig. 3. Period one (a) and two (b) operation of the buck converter.

where $x_{1}(t)=v(t)$ and $x_{2}(t)=i(t)$. The vector normal to $h$ is $\left[\begin{array}{ll}1 & 0\end{array}\right]^{T}$ and the two vector fields before and after the switching are:

$$
\begin{aligned}
& \mathbf{f}_{+}(\mathbf{x}(t))=\left[\begin{array}{c}
x_{2}(t) / C-x_{1}(t) / R C \\
-x_{1}(t) / L
\end{array}\right], \\
& \mathbf{f}_{-}(\mathbf{x}(t))=\left[\begin{array}{c}
x_{2}(t) / C-x_{1}(t) / R C \\
\left(V_{\text {in }}-x_{1}(t)\right) / L
\end{array}\right] .
\end{aligned}
$$

In that interval the monodromy matrix is given by:

$$
\mathbf{M}(T, 0)=\mathbf{\Phi}\left(T, t_{\Sigma}\right) \times \mathbf{S} \times \mathbf{\Phi}\left(t_{\Sigma}, 0\right)
$$

and the saltation matrix is given by ${ }^{1}$ :

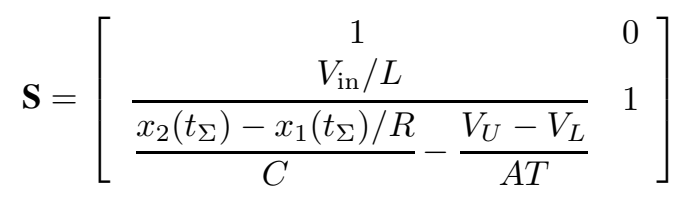

The state vector at $t=t_{\Sigma}$ can be found by numerically solving a nonlinear equation. Hence the monodromy matrix becomes:

$$
\mathbf{M}(T, 0)=e^{\mathbf{A}_{s} t_{\Sigma}} \times \mathbf{S} \times e^{\mathbf{A}_{s}\left(T-t_{\Sigma}\right)}
$$

Based on that it is possible to determine the stability of the limit cycle. The evolution of the Floquet multipliers is shown in Fig. 4 and hence it is clear that for $V_{i n}=24 \mathrm{~V}$ the system is stable while at $V_{i n}=25 \mathrm{~V}$ the system has a stable period two limit cycle.

\footnotetext{
${ }^{1}$ At this point we have to mention that there is one more saltation matrix at $t=T$. Since at the point $h$ is discontinuous the time derivative is infinite and hence the second saltation matrix is the identity matrix.
}

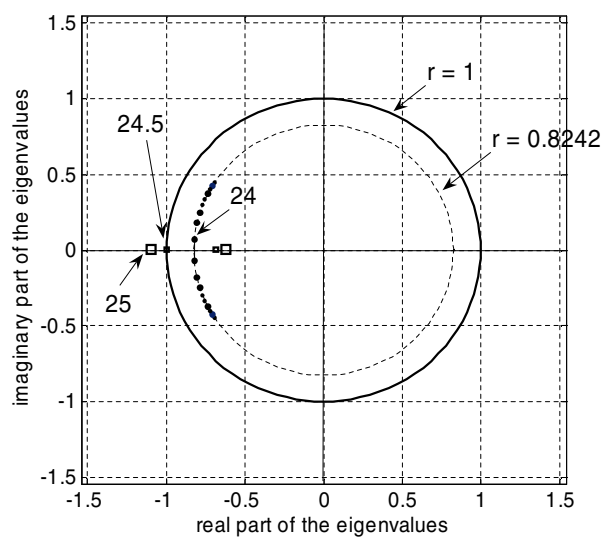

Fig. 4. Floquet multipliers, period 1. Squares indicate unstable system, solid circles stable system.

TABLE I

DUTY CYCLES OF PERIOD ONE, FOR TWO VALUES OF THE INPUT VOLTAGE

\begin{tabular}{|c|c|c|}
\hline$V_{i n}[\mathrm{~V}]$ & state vector & duty cycle \\
\hline 24 & {$[12.01390 .4861]^{T}$} & 0.5007 \\
\hline 25 & {$[12.02410 .4843]^{T}$} & 0.4813 \\
\hline
\end{tabular}

\section{PERIOD DOUBLING CONTROL}

\section{A. General concept}

Table I shows the duty cycle $\left(d=1-t_{\Sigma}\right)$ and the corresponding state vector for $V_{i n}=24$ and $V_{i n}=25 \mathrm{~V}$. It is clear from these values that the state vector at the instant of intersection does not greatly change. Hence the STM before and after the switching will not greatly alter. This implies that the main reason for the occurrence of the bifurcation is the change of the saltation matrix. So if we can reverse that change without greatly changing the duty cycle of the system we can stabilize the unstable period one orbit. To do that we have to carefully examine the structure of the saltation matrix (eqn. 4). Effectively $\mathbf{S}$ is determined by the two vector fields (which we cannot change) and by the derivative of $h$ with respect to time and by its derivative with respect to the state vector (normal).

\section{B. Buck converter - Period one}

1) Control based on injected sine wave: The system can be stabilized and the eigenvalues of the monodromy matrix pushed inside the unit circle by changing the value of $V_{\text {ref }}$ to $V_{\text {ref }}(1+a \sin (\omega t))$, where $\omega=2 \pi / T$ and the value of $a$ chosen to determine the desired location [6], [12]. The effect of this change can be seen by studying the time derivative of $h$ :

$$
\begin{gathered}
h(\mathbf{x}, t)=x_{1}-V_{\text {ref }}-V_{\text {ref }} a \sin \left(\omega d^{\prime} T\right)-\frac{V_{L}+\left(V_{U}-V_{L}\right) d^{\prime}}{A}=0 \Rightarrow \\
\frac{\partial h}{\partial t}=-\omega V_{\text {ref }} a \cos \left(\omega d^{\prime} T\right)-\frac{\left(V_{U}-V_{L}\right)}{A}
\end{gathered}
$$

where $d^{\prime}=1-d$.

Notice that $\sin (\omega t)$ at $t=d^{\prime} T$ is very small and hence the influence on $h$ will be very small. At the same time the value of $\cos (\omega t)$ will be large and will have a significant effect on the 
saltation matrix and hence the eigenvalues of the monodromy matrix.

It is obvious from eqn. (9) that by altering the value of $a$ it is possible to change the eigenvalues of the monodromy matrix. To optimally design the controller we can numerically solve the following nonlinear transcendental equation which will place the eigenvalues on the circle where the stable eigenvalues lie:

$$
\mid \operatorname{eig}(M(T, 0) \mid-0.82=0
$$

Based on that we derived a lookup table (Fig. 5) and from that a polynomial equation which determines the value of $a$ depending on the input voltage was derived:

$$
a\left(V_{i n}\right)=0.0001 V_{i n}^{2}+0.0037 V_{i n}+0.0328
$$

The corresponding response of the system for that controller for a sudden $50 \%$ increase in the load resistance when $V_{i n}=$ $26 \mathrm{~V}$ is shown in Fig. 6

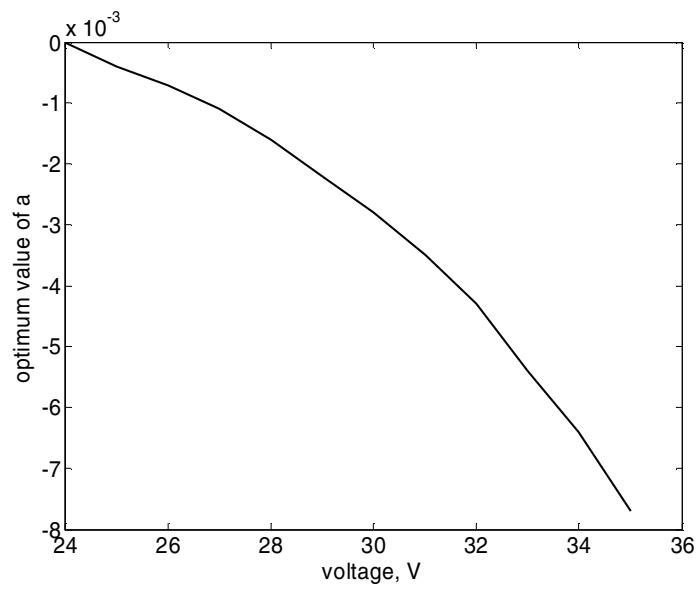

Fig. 5. Optimum values of $a$ for first controller.

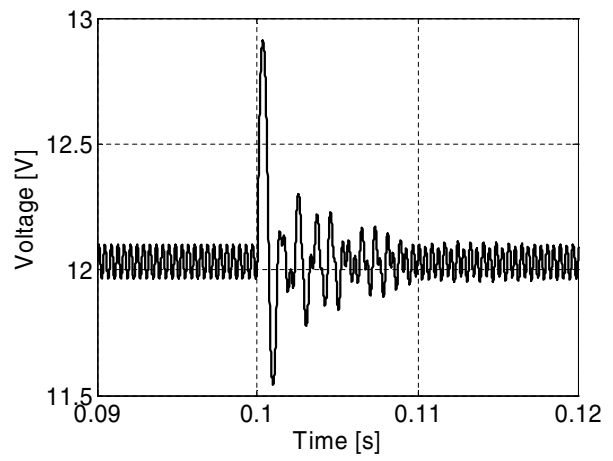

Fig. 6. Response of first controller, $V_{i n}=25 \mathrm{~V}$, Steady state error $=0.73 \mathrm{~V}$, Overshoot $=7.31 \%, t_{s}=0.018 \mathrm{~s}$.

2) Control based on signal proportional to the output voltage: Another control method [5] is based on changes of the normal of $h$. So we can slightly change the slope of $h$ to:

$h(\mathbf{x}(t), t)=(1+a) \times x_{1}(t)-V_{\text {ref }}-\frac{V_{L}+\left(V_{U}-V_{L}\right) t / T}{A}=0$
Giving a normal vector:

$$
\mathbf{n}=\left[\begin{array}{c}
1+a \\
0
\end{array}\right]
$$

and saltation matrix:

$$
\mathbf{S}=\left[\begin{array}{cc}
1 & 0 \\
\frac{(1+a) V_{\mathrm{in}} / L}{(1+a) \frac{x_{2}\left(t_{\Sigma}\right)-x_{1}\left(t_{\Sigma}\right) / R}{C}-\frac{V_{U}-V_{L}}{A T}} & 1
\end{array}\right]
$$

Instead of choosing the values of $a$ by examination [6] an optimum controller maybe obtained by placing the eigenvalues on a circle of radius 0.82 which is the radius where the eigenvalues are of the stable period one limit cycle.

$$
\mid \operatorname{eig}(M(T, 0) \mid-0.82=0
$$

The results are shown in Fig 7 (the response of this controller is shown in Fig. 11b)

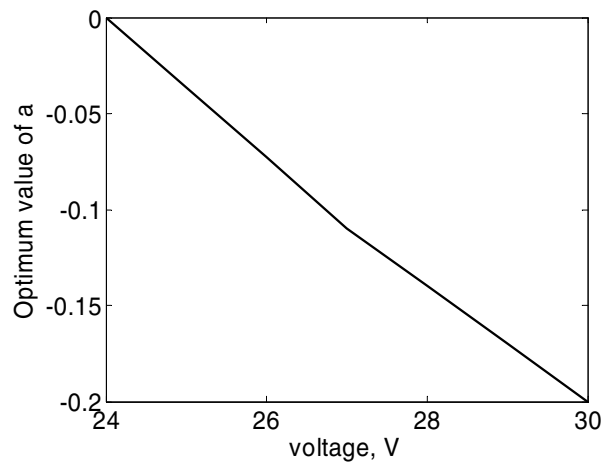

Fig. 7. Optimum values for the second controller

3) Control based on signal proportional to the output voltage and current: In this part of the paper we propose a new controller based on the values of the output voltage and the inductor current, giving a controller with two degrees of freedom. The switching manifold $h$ is changed to

$h(\mathbf{x}(t), t)=(1+a) x_{1}(t)+b x_{2}(t)-V_{\text {ref }}-\frac{V_{L}+\left(V_{U}-V_{L}\right) t / T}{A}$

This will change the normal to $\mathbf{n}^{T}=\left[\begin{array}{ll}1+a & b\end{array}\right]$ and hence the saltation matrix will be

$$
\mathbf{S}=\mathbf{I}+\frac{\left[\begin{array}{cc}
0 & 0 \\
(1+a) V_{i n} / L & b V i n / L
\end{array}\right]}{(1+a)\left(\frac{R x_{2}\left(t_{\Sigma}\right)-x_{1}\left(t_{\Sigma}\right)}{R C}\right)-b x_{1}\left(t_{\Sigma}\right) / L}-\frac{V_{U}-V_{L}}{A T}
$$

Now the monodromy matrix will be a function of two variables and we can appropriately tune one to stabilize the system and the other to decrease the settling time or the maximum overshoot of the system or even to minimize the error. Fig. 8 shows the maximum absolute value of the Floquet multiplier when $b=0$ for various values of $a$. It is clear that it is possible to place the eigenvalues of the system to almost any location in the unit circle. Fig. 9 shows the maximum absolute eigenvalue for variation of $b$ with $a$ fixed at -0.035 and -0.037 and $V_{i n}=25 \mathrm{~V}$. It is clear that by introducing a negative value of $b$ we moved the eigenvalues closer to the 
TABLE II

RESULTS OF THIRD CONTROLLER, $a=-0.037, V_{\text {in }}=25 \mathrm{~V}$

\begin{tabular}{|c|c|c|c|}
\hline$b$ & Error & Overshoot & Settling time \\
\hline-0.2 & 1.243 & $8.305 \%$ & $0.016 \mathrm{~s}$ \\
\hline 0 & 1.179 & $7.211 \%$ & $0.0052 \mathrm{~s}$ \\
\hline 0.1 & 1.147 & $7.059 \%$ & $0.0012 \mathrm{~s}$ \\
\hline
\end{tabular}

marginal stable case. To get a clearer picture of the influence of the two variables, Fig. 10 shows the maximum absolute eigenvalue for a series of values of $a$ and $b$.

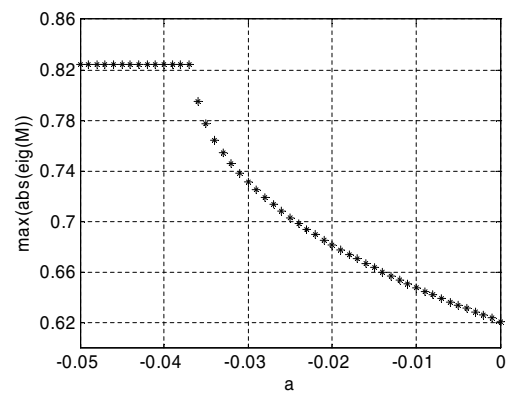

Fig. 8. Maximum absolute eigenvalue for $b=0$.
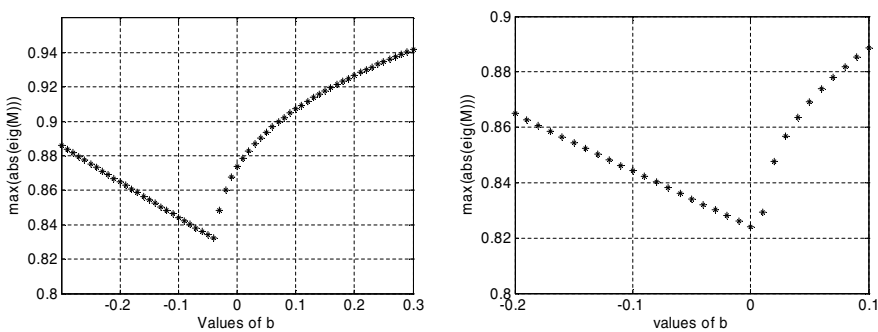

Fig. 9. Maximum absolute eigenvalue for $a=-0.035$ and -0.037 . The turnaround is because at some point the second eigenvalue becomes bigger than the first one

Fig. indicates that the introduction of $b$ moves the eigenvalues closer to the marginally stable case which adversely effects the overall control strategy, this pattern is stronger when $b$ is positive. However, an examination of Fig. 11 shows that negative values of $b$ make the system slower with more oscillations while positive values reduce the steady state error and increases the speed of the system (Table II). To derive the settling time, after the resistance increase, we let the system to settle down and we found the mean value of the sampled voltage (Poincaré map). Then we found the samples that are in a band of $\pm 2 \%$ and if all the successive samples are in this band then we recorded this time instant. The calculation of the steady state error was done by finding the maximum and minimum values of the voltage during the last cycles long after the resistance increase. The mean value was used as a steady state measurement and hence the error was calculated by finding the absolute value of the difference between the demanded voltage $\left(V_{\text {ref }}=11.3 \mathrm{~V}\right)$ and this mean value. It is believed that by further increasing the value of $b$ we can further minimize the error but for big values of $b$ we faced various numerical problems.

The comparison of Figs. 11 and 6 reveals that both con-

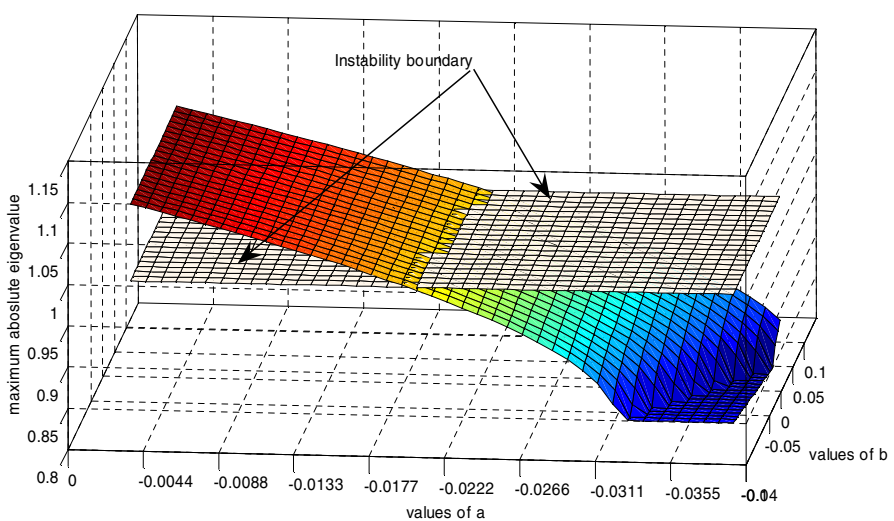

Fig. 10. Maximum absolute eigenvalue for $a \in[-0.04,0]$ and $b \in$ $[-0.1,0.1]$.
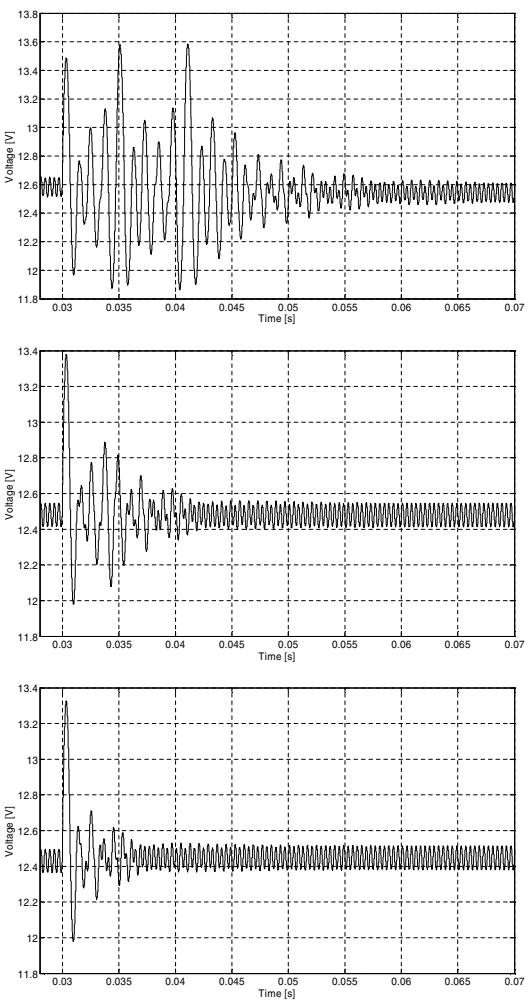

Fig. 11. Response of the system for $a=-0.037$ and $b=-0.2,0$ and 0.1, $V_{\text {in }}=25 \mathrm{~V}$

trollers give good results however, the controller which is based on an injected sine wave signal has smaller steady state error despite its comparative simplicity.

\section{Buck converter - Period two}

As with the control of the period one orbit it is possible to stabilize the period two or any other limit cycle that we desire. To demonstrate this capability of the proposed control strategy we are going to employ the first controller based on the injection of a sine wave signal due to its simplicity and superior results.

For the period two cycle the monodromy matrix is: $\mathbf{M}(2 T, 0)=\boldsymbol{\Phi}\left(2 T, t_{\Sigma_{3}}\right) \times \mathbf{S}_{3} \times \boldsymbol{\Phi}\left(t_{\Sigma_{3}}, T\right) \times \mathbf{S}_{2} \times \boldsymbol{\Phi}\left(T, t_{\Sigma_{1}}\right) \times$ $\mathbf{S}_{1} \times \mathbf{\Phi}\left(t_{\Sigma_{1}}, 0\right)$. The value of $\mathbf{S}_{2}$ is the identity matrix as at 
$t=T, h$ is discontinuous. The other two saltation matrices are:

$$
\mathbf{S}_{1}=\left[\begin{array}{cc}
1 & 0 \\
s_{a} & 1
\end{array}\right]
$$

where

$s_{a}=\frac{V_{\mathrm{in}} / L}{\frac{x_{2}\left(t_{\Sigma_{1}}\right)-x_{1}\left(t_{\Sigma_{1}}\right) / R}{C}-V_{\text {ref }} a \omega \cos \left(\omega t_{\Sigma_{1}}\right)-\frac{V_{U}-V_{L}}{A T}}$

and

$$
\mathbf{S}_{3}=\left[\begin{array}{cc}
1 & 0 \\
s_{b} & 1
\end{array}\right]
$$

where

$$
s_{b}=\frac{V_{\mathrm{in}} / L}{\frac{x_{2}\left(t_{\Sigma_{3}}\right)-x_{1}\left(t_{\Sigma_{3}}\right) / R}{C}-V_{\text {ref }} a \omega \cos \left(\omega t_{\Sigma_{3}}\right)-\frac{V_{U}-V_{L}}{A T}}
$$

This time the supervising controller is required to place the eigenvalues of the system on a circle of a radius 0.6792 which is the location of the eigenvalues of the stable period two cycle. Hence

$$
\mid \operatorname{eig}(M(2 T, 0) \mid-0.6792=0
$$

Resulting values are shown in Fig. 12. Fig. 13 shows the response of the controller. At $t=0.1 \mathrm{~s} V_{i n}$ is changed from $31 \mathrm{~V}$ to $32 \mathrm{~V}$ which will lead to a period four stable orbit. At $t=0.14 \mathrm{~s}$ the load resistance is increased by $50 \%$ again resulting in a stable period two orbit after an initial transient.

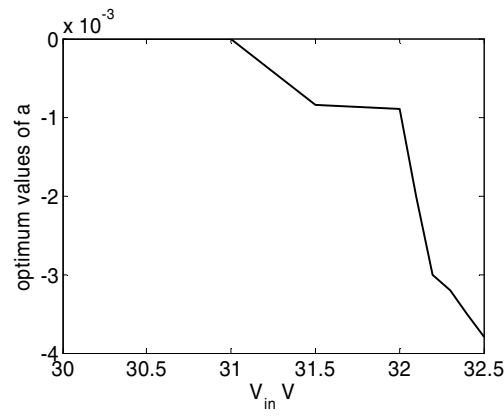

Fig. 12. Optimum values for the period 2 controller

\section{Boost converter}

To demonstrate the power of this method we apply the same controller to control a period one limit cycle in the currentmode controlled boost converter. We recognize the fact that usually the stable period one region of the boost converter is extended by using a slope compensator but we want to demonstrate that the method that we propose here is not only applicable to one converter but can be applied to almost any piecewise system.

In the case of the boost converter the bifurcation variable is the $I_{\text {ref }}$ and the switching manifold: $h(\mathbf{x}, t)=x_{2}-I_{\text {ref }}$,

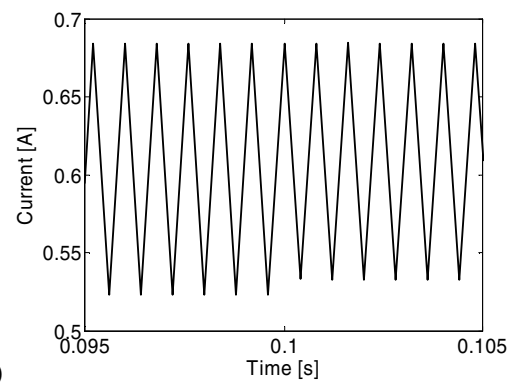

(a)

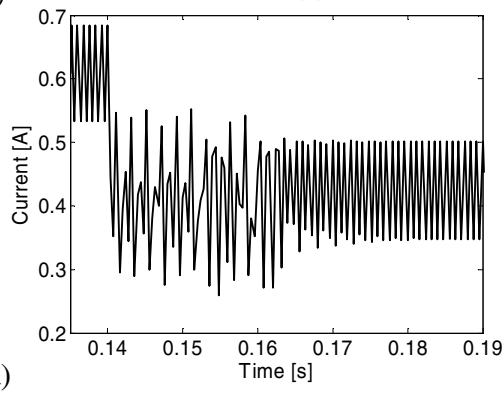

Fig. 13. Sampled response of the period 2 controller

where $x_{1}$ is the output voltage and $x_{2}$ is the inductor current. The two vector fields are:

$$
\mathbf{f}_{-}(x)=\left[\begin{array}{c}
-x_{1} / R C \\
V_{i n} / L
\end{array}\right], \quad \mathbf{f}_{+}(x)=\left[\begin{array}{c}
\frac{x_{2} R-x_{1}}{R C} \\
\frac{V_{i n}-x_{1}}{L}
\end{array}\right]
$$

The controller will change the reference current from $I_{r e f}$ to $a \times I_{\text {ref }} \times \sin (\omega t)$

Hence the resulted saltation matrix is given by:

$$
\begin{gathered}
\mathbf{S}=\mathbf{I}+\frac{\left(\mathbf{f}_{+}-\mathbf{f}_{-}\right) \mathbf{n}^{T}}{\mathbf{n}^{T} \mathbf{f}_{-}+\left.\frac{\partial h}{\partial t}\right|_{t=t_{\Sigma}}}= \\
{\left[\begin{array}{cc}
1 & \frac{x_{2}\left(t_{\Sigma}\right)}{C\left(\frac{V_{\text {in }}}{L}-I_{\text {ref }} a \omega \cos \left(\omega t_{\Sigma}\right)\right)} \\
0 & \left.1-\frac{x_{1}\left(t_{\Sigma}\right)}{L\left(\frac{V_{i n}}{L}+-I_{r e f} a \omega \cos \left(\omega t_{\Sigma}\right)\right.}\right)
\end{array}\right]}
\end{gathered}
$$

Instead of

$$
\mathbf{S}=\mathbf{I}+\frac{\left(\mathbf{f}_{+}-\mathbf{f}_{-}\right) \mathbf{n}^{T}}{\mathbf{n}^{T} \mathbf{f}_{-}}=\left[\begin{array}{cc}
1 & \frac{x_{2}\left(t_{\Sigma}\right)}{V_{i n}} \\
& C \frac{x_{1}\left(t_{\Sigma}\right)}{V_{i n}}
\end{array}\right]
$$

By numerically solving

$$
\mid \operatorname{eig}(M(T, 0) \mid-0.997=0
$$

we got the following values of $a$ Fig. 14. The location of the eigenvalues was chosen to avoid high values of $a$ resulting in a significant change in the shape of the period two limit cycle.

The sampled response of the controller is shown in Fig. 15. At $t=0.1$ s we change the reference current from 0.49 A to 


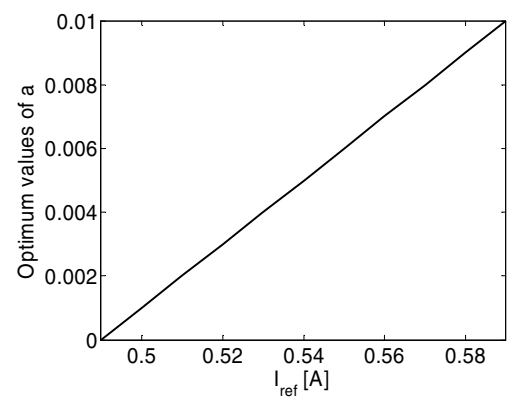

Fig. 14. Optimum values of a, boost converter

$0.51 \mathrm{~A}$ and then at $t=0.105 \mathrm{~s}$ we increased the load resistance by $50 \%$.

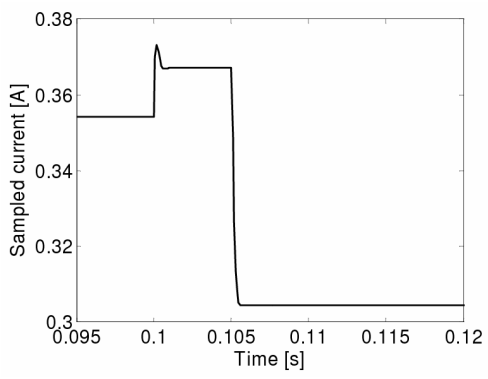

Fig. 15. Response of the boost converter under the period doubling controller

\section{DISCUSSION}

The results presented in this paper show that all three controllers are easy to implement and produce good results which greatly extend the desired periodic pattern for a big range of the bifurcation parameter. The comparison clearly shows that the sine wave based controller produces the best results with smaller steady state error, smaller settling time and an overshoot of less than $9 \%$ when the resistive load suddenly increases by $50 \%$. Another advantage of this controller is that it does not require the information of the state vector and is only dependent on the bifurcation variable. This makes it extremely attractive especially for apparitions using the current controlled boost converter since the bifurcation variable can be the demanded current which as chosen by the user. The response of the second controller is also satisfactory and it does not require any extra sensors since the output voltage is used anyway for the feedback controller. However, there is a larger steady state error and a longer transient period. The addition of the current signal in the supervising controller (third controller) improved the response of the system, decreasing both settling time and overshoot but it has the disadvantage of needing an extra sensor to measure the inductor current. This is a problem especially for the buck converter which usually does not require a current control loop as it is a minimum phase system [13]. Combination of different controllers have also been tried but the improvements where not sufficient to justify the increased complexity.

\section{CONCLUSIONS}

A new, versatile control method has been proposed that can be applied to any power electronic switched system stabilize to stabilize any periodic orbit. We have demonstrated the universality of the method by suing it to control various limit cycles for the step up and down converters. It is clear that this method can easily be adopted for practical use as they provide good results without any extra complicated bifurcation or chaotic control law. In all cases the system is robust, with small steady state error and small settling time and maximum overshot.

\section{APPENDIX}

Parameters of buck converter: $V_{\text {in }}=24 V, V_{\text {ref }}=11.3 \mathrm{~V}$, $L=20 \mathrm{mH}, R=22 \Omega, C=47 \mu \mathrm{F}, A=8.4, T=1 / 2500 \mathrm{~s}$, the ramp signal varies from $3.8 \mathrm{~V}$ to $8.2 \mathrm{~V}$.

Parameters of boost converter: $C=10 \mu F, L=1.5 \mathrm{mH}$, $R=40 \Omega, T=100 \mu s$ and $V_{i n}=5 \mathrm{~V}$.

\section{REFERENCES}

[1] Y. Ma, H. Kawakami, and C. Tse, "Bifurcation analysis of switched dynamical systems with periodically moving borders," IEEE Transactions on Circuits and Systems-I, vol. 51, pp. 1184-1193, June 2004.

[2] E. Fossas and G. Olivar, "Study of chaos in the buck converter," IEEE Transactions on Circuits and Systems-I, vol. 43, no. 1, pp. 13-25, 1996.

[3] K. Chakrabarty, G. Poddar, and S. Banerjee, "Bifurcation behaviour of the buck converter," IEEE Transactions on Power Electronics, vol. 11, pp. 439-447, May 1996.

[4] S. Banerjee and G. C. Verghese, eds., Nonlinear Phenomena in Power Electronics: Attractors, Bifurcations, Chaos, and Nonlinear Control. New York, USA: IEEE Press, 2001.

[5] D. Giaouris, S. Banerjee, B. Zahawi, and V. Pickert, "Stability analysis of power electronic dc-dc converters using complete-cycle solution matrix," Submitted to: IEEE Transactions on Circuits and Systems - I.

[6] D. Giaouris, A. Elbkosh, V. Pickert, B. Zahawi, and S. Banerjee, "Control of period doubling bifurcations in dc-dc converters," in International Conference Control 2006, (Glasgow - Scotland), 2006.

[7] Y. A. Kuznetsov, Elements of Applied Bifurcation Theory. Springer, 2004.

[8] R. I. Leine and H. Nijmeijer, Dynamics and Bifurcations of Non-Smooth Mechanical Systems. Springer, 2004.

[9] R. I. Leine, D. H. V. Campen, and B. L. V. de Vrande, "Bifurcations in nonlinear discontinuous systems," Nonlinear Dynamics, vol. 23, pp. 105-164, 2000.

[10] M. Di Bernardo and Francesco Vasca, "Discrete-time maps for the analysis of bifurcations and chaos in dc/dc converters," IEEE Transactions on Circuits and Systems-I, vol. 47, no. 2, pp. 130-143, 2000.

[11] M. Di Bernardo, F. Garofalo, L. Glielmo, and F. Vasca, "Switchings, bifurcations and chaos in DC-DC converters," IEEE Transactions on Circuits and Systems-I, vol. 45, no. 2, pp. 133-141, 1998.

[12] Y. Zhou, C. K. Tse, S. S. Qiu, and F. C. M. Lau, "Applying resonant parametric perturbarion to control chaos in the buck dc/dc converter with phase shift and frequency mismatch considerations," International Journal of Bifurcation and Chaos, vol. 13, pp. 3459-3471, November 2003.

[13] C. K. Tse, Complex Behavior of Switching Power Converters. Boca Raton, USA: CRC Press, 2003. 